\title{
Budget impact analysis of trilaciclib for decreasing the incidence of chemotherapy- induced myelosuppression in patients with extensive-stage small cell lung cancer in the United States
}

Ivo Abraham, PhD; Amit Goyal, MBA; Baris Deniz, MSc; Donald Moran, PharmD; Marc Chioda, PharmD; Karen M MacDonald, PhD; Huan Huang, PhD

\section{What is already known about this subject}

- Chemotherapy-induced myelosuppression, which commonly manifests as neutropenia, anemia, and/or thrombocytopenia, is a frequent and severe complication of treatment observed in patients with extensive-stage small cell lung cancer (ES-SCLC).

- Myelosuppression places a substantial burden on patients with ES-SCLC receiving chemotherapy.

- Conventional interventions for the management of myelosuppression are specific to single hematopoietic lineages.

\section{What this study adds}

- Trilaciclib is the first therapy shown to protect multiple hematopoietic lineages simultaneously, thereby protecting the bone marrow of adult patients with ES-SCLC from chemotherapy-induced damage.

- The acquisition cost associated with the addition of trilaciclib is estimated to be offset by a reduction in myelosuppressive adverse events and their management costs.

- The financial budget impact is estimated to be a net cost saving to health plans.

\begin{abstract}
Author affiliations
Ivo Abraham, PhD, Center for Health Outcomes and PharmacoEconomic Research and Department of Pharmacy Practice and Science, College of Pharmacy, University of Arizona, Tucson, and University of Arizona Cancer Center, Tucson. Ivo Abraham, PhD, and Karen M MacDonald, PhD, Matrix45, Tucson, AZ. Amit Goyal, MBA, ZS Associates, Gurgaon, India, and Baris Deniz, MSc, ZS Associates, New York, NY. Donald Moran, PharmD; Marc Chioda, PharmD; and Huan Huang, PhD, G1 Therapeutics, Inc, Research Triangle Park, NC.
\end{abstract}

AUTHOR CORRESPONDENCE: Ivo Abraham, 520.626.4425;

abraham@pharmacy.arizona.edu

J Manag Care Spec Pharm 2022;28(4):435-48

Copyright $\odot 2022$, Academy of Managed Care Pharmacy. All rights reserved.

OBJECTIVE: To estimate the budget impact of administering trilaciclib prior to chemotherapy to manage chemotherapy-induced myelosuppression in adults with ES-SCLC from a US payer perspective.

METHODS: A budget impact model was developed to assess the impact of introducing trilaciclib to a hypothetical 
1 million-member health insurance plan. The model compared 2 market scenarios: a current scenario of standard treatments for ES-SCLC without trilaciclib, and an alternative scenario of standard treatment plus trilaciclib. Population, clinical, and cost inputs were derived from published literature and trilaciclib clinical trial data. Model outcomes included the number of myelosuppressive adverse events (AEs), costs of treatment, costs of $A E$ management, total cost, and per-member per-month (PMPM) costs. The budget impact of trilaciclib was calculated as the difference in cost (2021 US dollars) between the 2 scenarios over a 1- to 5 -year time horizon. Scenario and deterministic sensitivity analyses were conducted to assess uncertainty around key model inputs.

RESULTS: An estimated total of 301 patients were eligible for treatment with trilaciclib over a 5 -year period. The use of trilaciclib was estimated to reduce the number of myelosuppressive AEs over a 5 -year period (events avoided included 108 for neutropenia, 7 for febrile neutropenia, 23 for anemia, and 46 for thrombocytopenia) compared with the scenario without trilaciclib. The adoption of trilaciclib was associated with a cost saving of \$801,254 (\$0.013 PMPM) over 5 years. The acquisition cost for trilaciclib $(\$ 3,704,199)$ was offset by the reduction in AE management cost $(\$ 4,282,748)$ and reduction in prophylactic granulocyte colony-stimulating factor use $(\$ 222,704)$. The cost savings associated with trilaciclib began in year 1 (total \$34,388; \$0.003 PMPM) and accrued over time.

CONCLUSIONS: The acquisition cost of trilaciclib is projected to be offset by a reduction in the costs of managing AEs related to myelosuppression when added to standard chemotherapy regimens for ES-SCLC. The net budget impact of trilaciclib is estimated to be a cost saving.

Lung cancer is the second most common cancer in both men and women and the leading cause of cancer-related mortality in the United States. ${ }^{1}$ Non-small cell lung cancer (NSCLC) is most common ( $>80 \%$ of lung cancer diagnoses), whereas small cell lung cancer (SCLC) comprises approximately $13 \%-17 \%$ of all lung cancer diagnoses. ${ }^{2-4}$ SCLC is the most aggressive form of lung cancer and is characterized by poor prognosis, with a 5-year survival rate of $7 \%$ compared with $25 \%$ for NSCLC. ${ }^{2}$ Early diagnosis of SCLC is difficult, owing to limited specific symptoms, rapid tumor growth, and early, widespread metastases., ${ }^{4,5}$ Consequently, approximately two-thirds of patients with SCLC have extensive-stage (ES) disease at diagnosis, requiring systemic treatment. ${ }^{4}$

Standard first-line treatment for ES-SCLC comprises a core platinum/etoposide combination chemotherapy regimen, with or without the addition of atezolizumab or durvalumab. ${ }^{6,7}$ Although SCLC appears to be responsive to initial treatment, most patients relapse within 6 months ${ }^{6,7}$; median progression-free survival is approximately 5 months and median overall survival may reach 12-13 months with further systemic therapy. ${ }^{8,9}$ Historically, topotecan was the preferred second-line treatment; however, other recommended second-line treatment options for those who relapse within 6 months now include lurbinectedin, irinotecan, gemcitabine, paclitaxel, and docetaxel.,10,11

Chemotherapy-induced myelosuppression (CIM), which commonly exhibits as neutropenia, anemia, and/or thrombocytopenia, is a frequent complication of treatment observed in patients with ES-SCLC. ${ }^{12-15}$ The incidence of grade 3/4 neutropenia, anemia, and thrombocytopenia in clinical trials of first-line platinum/etoposide with or without immuno-oncology therapy has been reported to span $23 \%-48 \%, 9 \%-22 \%$, and $6 \%-20 \%$, respectively. ${ }^{8,9,16,17}$ Corresponding incidence rates among clinical trial participants treated with second-line topotecan have been reported at $54 \%-70 \%, 31 \%-42 \%$, and $29 \%-54 \%$, respectively. ${ }^{10,18}$ These myelosuppressive adverse events (AEs) are associated with an increased risk of morbidity, oftentimes experienced as fatigue, infection, and sepsis, ${ }^{19}$ as well as increased mortality, poorer health-related quality of life, and greater utilization of health care services that incur additional costs. ${ }^{20-25}$

A substantial economic burden is associated with chemotherapy-induced myelosuppressive AEs in the United States. ${ }^{26-28}$ Hospitalizations due to neutropenia account for $8.3 \%$ of all cancer-related hospitalization costs for adults in the United States and have an attributable estimated cost of \$2.3 billion per year (2012 US dollars [USD], \$3.0 billion in 2021 USD). ${ }^{27,29}$ Meanwhile, mean health care costs attributable to anemia have ranged from \$22,775 (2006 USD; \$35,547 in 2021 USD) to \$93,454 (2006 USD; \$145,861 in 2021 USD) per patient per year. ${ }^{26,29}$ The mean cost estimates for thrombocytopenia have extended from \$1,395 (2006 USD; $\$ 2,177$ in 2021 USD) per chemotherapy cycle to $\$ 36,448$ (2016 USD; \$41,248 in 2021 USD) per episode. ${ }^{26,28-30}$

Current options for the management of CIM are specific to single hematopoietic lineages. These options include granulocyte colony-stimulating factor (G-CSF) for the prevention or management of neutropenia; red blood cell transfusions, administration of erythropoiesis-stimulating agents, and iron supplementation for anemia; and platelet transfusion and antifibrinolytic agents for thrombocytopenia. ${ }^{23,31-33}$ However, as these approaches do not protect the bone marrow from chemotherapy-induced cytotoxic effects and impart their own risks for adverse reactions, they can be considered suboptimal. ${ }^{34}$ CIM may lead to dose delays, dose reductions, or discontinuation of chemotherapy. Clinicians have suggested that the delivery of optimal chemotherapy dose intensity and duration should be considered a major quality indicator in the care of 


\section{FIGURE 1 Model Structure}

Population selection
Number of members in health plan

Incidence of ES-SCLC

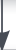

$\downarrow$

Proportion of patients treated with systemic chemotherapy

Formulary distribution

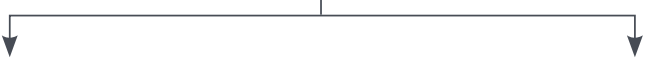

Without trilaciclib (current scenario)

With trilaciclib (alternative scenario)

Market share of treatment regimens

Market share of treatment regimens

Resource utilization and costs

AEs and management of AEs

AEs and management of AEs

Drug costs and other costs

Budget impact

Total cost without trilaciclib
Drug costs and other costs

Total cost with trilaciclib

Budget impact of trilaciclib (difference)

${ }^{a}$ Expressed as total annual costs and costs per-member per-month. $A E=$ adverse event $E S-S C L C=$ extensive-stage small cell lung cancer

patients with curable cancers, ${ }^{35-38}$ as treatment interruption may subsequently impact tumor control and patient outcomes. ${ }^{39-41}$

Trilaciclib (COSELA, G1 Therapeutics, Inc.), a firstin-class, intravenous (IV) cyclin-dependent kinase 4/6 inhibitor, transiently arrests hematopoietic stem and progenitor cells in the G1 phase of the cell cycle, thereby providing multilineage protection from the cytotoxic effects of chemotherapy. ${ }^{34,42}$ Trilaciclib is administered as a 30-minute IV infusion completed within 4 hours prior to the start of chemotherapy on each day chemotherapy is administered. The myeloprotective effects of trilaciclib have been demonstrated in 3 phase 2 clinical trials of patients with treatment-naive or previously treated ES-SCLC. ${ }^{43-45}$ In each trial, patients received trilaciclib or placebo prior to standard chemotherapy (study G1T28-05: first-line carboplatin, etoposide, and atezolizumab; study G1T28-02: first-line carboplatin and etoposide; study G1T28-03: second-/thirdline topotecan). All 3 trials allowed G-CSF use except for primary prophylactic use in cycle 1 ; therapeutic G-CSF use was allowed in all cycles. Administering trilaciclib prior to chemotherapy significantly reduced the duration of grade 4 neutropenia in cycle 1 and occurrence of grade 4 neutropenia during the treatment period (dual primary endpoints) and numerically improved key secondary endpoints, including occurrence of grade $\geq 3$ hematologic laboratory abnormalities. 


\section{TABLE 1 Model Inputs}

\begin{tabular}{|c|c|c|}
\hline Input & $\begin{array}{c}\text { Base-case } \\
\text { estimate }\end{array}$ & Sources \\
\hline \multicolumn{3}{|l|}{ Population, \% } \\
\hline Incidence of lung cancer & 0.07 & de Groot 2018, Worldometers 201949,50 \\
\hline Proportion of patients with SCLC (within lung cancer population) & 15.0 & Wang $2017^{3}$ \\
\hline Proportion of patients with ES-SCLC (within SCLC population) & 66.0 & American Cancer Society ${ }^{51}$ \\
\hline Proportion of patients treated with first-line chemotherapy & 90.0 & $\begin{array}{l}\text { Data on file 2015, Decision Resources Group, Small Cell Lung } \\
\text { Cancer - Landscape \& Forecast }\end{array}$ \\
\hline $\begin{array}{l}\text { Proportion of patients treated with first-line chemotherapy and } \\
\text { eligible to receive trilaciclib }\end{array}$ & 72.2 & $\begin{array}{l}\text { Data on file 2019, Kantar Health, CancerMPact Treatment } \\
\text { Architecture SCLC USA }\end{array}$ \\
\hline $\begin{array}{l}\text { Proportion of patients treated in first line who will be treated with } \\
\text { second-line chemotherapy within the same year and eligible to } \\
\text { receive trilaciclib }\end{array}$ & 26.3 & $\begin{array}{l}\text { Data on file 2019, Kantar Health, CancerMPact Treatment } \\
\text { Architecture SCLC USA }\end{array}$ \\
\hline $\begin{array}{l}\text { Proportion of patients achieving long-term remission on first-line } \\
\text { therapy }\end{array}$ & 14.6 & $\begin{array}{l}\text { Data on file 2019, Kantar Health, CancerMPact Treatment } \\
\text { Architecture SCLC USA }{ }^{a}\end{array}$ \\
\hline $\begin{array}{l}\text { Proportion of patients achieving long-term remission on } \\
\text { second-line therapy }\end{array}$ & 11.3 & $\begin{array}{l}\text { Data on file 2019, Kantar Health, CancerMPact Treatment } \\
\text { Architecture SCLC USA }{ }^{a}\end{array}$ \\
\hline $\begin{array}{l}\text { Proportion of patients with long-term remission re-treated } \\
\text { (first- or second-line therapy) with systemic chemotherapy }\end{array}$ & $51.3^{\mathrm{b}}$ & Shiozawa $2018^{69}$ \\
\hline
\end{tabular}

\section{Market share, \%}

First-line chemotherapy regimen

\begin{tabular}{|c|c|c|}
\hline Etoposide/carboplatin/atezolizumab & 68.0 & \multirow{3}{*}{ G1 Therapeutics market research } \\
\hline Etoposide/carboplatin & 20.0 & \\
\hline Etoposide/cisplatin & 12.0 & \\
\hline \multicolumn{3}{|l|}{ Second-line chemotherapy regimen } \\
\hline Topotecan & 73.0 & \multirow{3}{*}{ G1 Therapeutics market research } \\
\hline Etoposide/carboplatin & 19.0 & \\
\hline Etoposide/cisplatin & 8.0 & \\
\hline \multicolumn{3}{|c|}{ Trilaciclib uptake in patients eligible for first-line therapy (alternative scenario) } \\
\hline Year 1 & 6.6 & \multirow{5}{*}{ G1 Therapeutics market research } \\
\hline Year 2 & 21.7 & \\
\hline Year 3 & 32.0 & \\
\hline Year 4 & 39.0 & \\
\hline Year 5 & 43.6 & \\
\hline
\end{tabular}

Trilaciclib uptake in patients eligible for second-line therapy (alternative scenario)

\begin{tabular}{|c|c|c|}
\hline Year 1 & 6.9 & \multirow{5}{*}{ G1 Therapeutics market research } \\
\hline Year 2 & 22.3 & \\
\hline Year 3 & 32.9 & \\
\hline Year 4 & 40.0 & \\
\hline Year 5 & 44.8 & \\
\hline \multicolumn{3}{|l|}{ Prophylactic G-CSF } \\
\hline Prophylactic use of G-CSF without trilaciclib, \% & 26.0 & G1 Therapeutics market research \\
\hline Reduction in use of prophylactic G-CSF with trilaciclib, \% & $50.0^{\mathrm{b}, \mathrm{c}}$ & Weiss $2021^{55}$ \\
\hline Average G-CSF cost (WAC plus administration cost) per cycle, \$ & 5,733 & Drugs.com 2020, Eldar-Lissai $2008^{53,56}$ \\
\hline Average number of prophylactic G-CSF cycles & $3.41^{\mathrm{b}}$ & Naeim $2013^{57}$ \\
\hline
\end{tabular}




\section{TABLE 1 Model Inputs (continued)}

\begin{tabular}{|c|c|c|}
\hline Input & $\begin{array}{l}\text { Base-case } \\
\text { estimate }\end{array}$ & Sources \\
\hline \multicolumn{3}{|l|}{ Grade 3/4 AE management cost, \$ } \\
\hline Neutropenia & $21,089^{b, d}$ & Wong $2018^{30}$ \\
\hline Febrile neutropenia & $22,563^{b, d}$ & Weycker $2015^{21}$ \\
\hline Anemia & $24,868^{\mathrm{b}, \mathrm{d}}$ & Wong $2018^{30}$ \\
\hline Thrombocytopenia & $27,860^{b, d}$ & Wong $2018^{30}$ \\
\hline \multicolumn{3}{|l|}{ Drug costs, $\$$} \\
\hline \multicolumn{3}{|l|}{ First-line chemotherapy (cost per regimen) } \\
\hline Etoposide/carboplatin/atezolizumab & $49,538^{\mathrm{e}}$ & \multirow{3}{*}{$\begin{array}{l}\text { National Comprehensive Cancer Network 2021, Drugs.com } \\
\text { 2020, Duh 20137,53,70 }\end{array}$} \\
\hline Etoposide/carboplatin & $12,435^{\mathrm{e}}$ & \\
\hline Etoposide/cisplatin & $11,597^{\mathrm{d}}$ & \\
\hline \multicolumn{3}{|c|}{ Second-line chemotherapy (cost per regimen) } \\
\hline Topotecan & $20,803^{e}$ & \multirow{3}{*}{$\begin{array}{l}\text { National Comprehensive Cancer Network 2021, Drugs.com } \\
\text { 2020, Duh 20137,53,70 }\end{array}$} \\
\hline Etoposide/carboplatin & $12,435^{e}$ & \\
\hline Etoposide/cisplatin & $11,597^{\mathrm{e}}$ & \\
\hline Trilaciclib (WAC cost per cycle) & $8,502^{f}$ & International Business Machines $2021^{54}$ \\
\hline
\end{tabular}

${ }^{a}$ Based on online research with direct responses from oncologists.

${ }^{b}$ Computed from data reported in the sources.

cProphylactic G-CSF use with trilaciclib is $13.0 \%$ (50\% of $26 \%$ ) across all 5 years.

${ }^{d}$ Based on management costs for severe AEs (requiring inpatient stay).

eTotal cost including acquisition and administration costs plus accounting for the number of cycles and visits per cycle.

${ }^{\prime}$ Estimate based on a platinum-/etoposide-containing regimen (3 doses per cycle, $\$ 2,834$ per dose); for a topotecan-containing regimen, this was estimated as $\$ 14,170$ (5 doses per cycle, \$2,834 per dose).

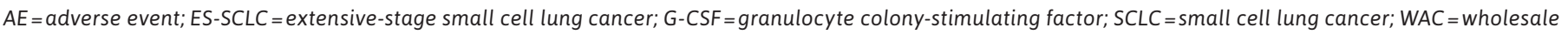
acquisition cost.

In February 2021, trilaciclib was approved by the US Food and Drug Administration to decrease the incidence of CIM in adult patients when administered prior to a platinum-/ etoposide-containing regimen or topotecan-containing regimen for ES-SCLC. ${ }^{46}$ As of March 2021, trilaciclib is recommended by the National Comprehensive Cancer Network (NCCN) guidelines for both SCLC and hematopoietic growth factors as a prophylactic option to decrease the incidence of CIM when administered before platinum/ etoposide plus or minus immune checkpoint inhibitorcontaining regimens or a topotecan-containing regimen for $\mathrm{ES}-\mathrm{SCLC}$. 733

In this study, a budget impact model (BIM) was developed to estimate the financial impact of prescribing trilaciclib for its approved indication.

\section{Methods}

\section{MODEL OVERVIEW}

The current BIM was developed following guidance on good modeling practices from the International Society for Pharmacoeconomic Outcomes and Research. ${ }^{47}$ Key elements included estimating an appropriate size of population, use of the current first- and second-line chemotherapy regimens with and without the new intervention, the most recent costs of treatment and management strategies, and incorporation of a sensitivity analysis.

A model was built using Microsoft Excel. The model time frame was up to 5 years, and the perspective was from a health care payer in the United States. The model created 2 scenarios to assess the budget impact of trilaciclib: a current scenario of standard treatments for ES-SCLC without trilaciclib and an alternative scenario of the same standard 


\section{TABLE 2}

Incidence and Frequency of Grade 3/4 Chemotherapy-Induced Myelosuppression AEs for Chemotherapy With or Without Trilaciclib

\begin{tabular}{|c|c|c|c|c|c|c|c|c|}
\hline \multirow[b]{2}{*}{ Chemotherapy } & \multicolumn{2}{|c|}{ Neutropenia } & \multicolumn{2}{|c|}{ Febrile neutropenia } & \multicolumn{2}{|c|}{ Anemia } & \multicolumn{2}{|c|}{ Thrombocytopenia } \\
\hline & $\begin{array}{l}\text { Without } \\
\text { trilaciclib }\end{array}$ & $\begin{array}{c}\text { With } \\
\text { trilaciclib }\end{array}$ & $\begin{array}{l}\text { Without } \\
\text { trilaciclib }\end{array}$ & $\begin{array}{c}\text { With } \\
\text { trilaciclib }\end{array}$ & $\begin{array}{l}\text { Without } \\
\text { trilaciclib }\end{array}$ & $\begin{array}{c}\text { With } \\
\text { trilaciclib }\end{array}$ & $\begin{array}{l}\text { Without } \\
\text { trilaciclib }\end{array}$ & $\begin{array}{c}\text { With } \\
\text { trilaciclib }\end{array}$ \\
\hline \multicolumn{9}{|c|}{ Incidence of grade 3/4 AEs with first-line treatment, $\%$} \\
\hline Etoposide/carboplatin/atezolizumab ${ }^{44}$ & 60 & 21 & 6 & 2 & 30 & 17 & 38 & 2 \\
\hline Etoposide/carboplatin ${ }^{43}$ & 68 & 11 & 8 & 3 & 19 & 5 & 8 & 8 \\
\hline Etoposide/cisplatin 13,a & 68 & 18 & 10 & 3 & 12 & 6 & 15 & 3 \\
\hline Average events/patient, $\mathrm{n}^{\mathrm{b}}$ & 2.5 & 1.3 & 1.3 & 1.0 & 1.6 & 1.6 & 1.7 & 1.5 \\
\hline \multicolumn{9}{|c|}{ Incidence of grade 3/4 AEs with second-line treatment, $\%$} \\
\hline Topotecan ${ }^{45}$ & 86 & 75 & 18 & 6 & 61 & 28 & 57 & 56 \\
\hline Etoposide/carboplatin 52, a & 20 & 17 & 6 & 2 & 21 & 10 & 31 & 30 \\
\hline Etoposide/cisplatin 13,a & 68 & 59 & 10 & 3 & 12 & 6 & 15 & 15 \\
\hline Average events/patient, $\mathrm{n}^{\mathrm{b}}$ & 3.3 & 2.5 & 1.2 & 1.0 & 1.8 & 1.7 & 4.1 & 2.5 \\
\hline
\end{tabular}

${ }^{a}$ Calculated from the AE rates reported for chemotherapy and by applying the relative risk reduction associated with trilaciclib (pooled average from G1T28-0544 and $\mathrm{G} 1 \mathrm{~T} 28-02^{43}$ clinical trials) to the rate of AEs without trilaciclib.

${ }^{b}$ Calculated as the total number of grade 3/4 AEs during the trial duration divided by the number of patients having $\geq 1$ grade $3 / 4$ AE

$A E=$ adverse event.

treatments in combination with trilaciclib (Figure 1). The difference in overall costs between the 2 scenarios was the budget impact driven by the introduction of trilaciclib.

Population inputs were based on published epidemiology data. Chemotherapies considered in the model were selected according to standard care. AE profiles of treatment regimens were derived from trilaciclib clinical trials and published literature. Drug costs were calculated from published wholesale acquisition costs (WACs) and included administration costs. The WAC was the only publicly available cost for trilaciclib at the time of model development (average selling price [ASP] was not yet available); therefore, WAC was used for all drugs in the model for consistency. The first ASP for trilaciclib was released in October 2021 and was subsequently incorporated in a scenario analysis, where the October 2021 Centers for Medicare \& Medicaid Services (CMS) Medicare Part B reimbursement limit based on second quarter (2Q) 2021 ASP was used for all drug acquisition cost inputs. ${ }^{48} \mathrm{AE}$ management costs were obtained from published literature. The total budget impact was calculated for the entire population of members in the hypothetical health plan, along with differences in permember per-month (PMPM) costs between the current and alternative scenarios. Scenario and deterministic sensitivity analyses (DSAs) were also conducted.

\section{MODEL INPUTS}

Eligible Population. The BIM started with a hypothetical US health insurance plan of 1 million members, which was subsequently reduced to concentrate only on those patients with ES-SCLC. The population estimates for ES-SCLC (incidence of lung cancer in the United States, proportion of patients with lung cancer who have SCLC, and proportion of patients with SCLC who have ES disease) were derived from published epidemiological data (Table 1), ${ }^{3,49-51}$

Estimates for patients with ES-SCLC who were eligible to receive trilaciclib, including those receiving first- or second-line chemotherapy, the proportion of chemotherapy-treated patients eligible for trilaciclib, and the proportions of patients achieving remission or retreated with chemotherapy after remission, were based on published literature and complemented by market research data (Table 1).

Treatments and Market Share. Within the model, the estimated market share of the chemotherapy regimens and trilaciclib uptake were based on G1 Therapeutics market research (Table 1). In the base case, etoposide/carboplatin/atezolizumab (68\%), etoposide/carboplatin (20\%), and etoposide/cisplatin (12\%) comprised the first-line chemotherapy regimens; and topotecan (73\%), etoposide/ carboplatin (19\%), and etoposide/cisplatin (8\%) comprised the second-line chemotherapy regimens (Supplementary 
Table 1, available in online article). Trilaciclib uptake was assumed to increase gradually over time, from $6.6 \%$ in the first-line setting and $6.9 \%$ in the second-line setting in year 1 , to $43.6 \%$ and $44.8 \%$ in year 5 , respectively (Table 1 ).

Number of Chemotherapy-Induced Myelosuppressive AEs. The incidence of chemotherapy-induced myelosuppressive AEs (neutropenia, febrile neutropenia [FN], anemia, and thrombocytopenia) was calculated using data from the trilaciclib clinical trials and published literature (Table 2). In the first-line setting, $\mathrm{AE}$ incidence for etoposide/carboplatin/atezolizumab or etoposide/carboplatin with and without trilaciclib was based on the trilaciclib G1T28-05 and G1T28-02 clinical trials, respectively. ${ }^{43,44} \mathrm{AE}$ rates for etoposide/cisplatin were based on published literature..$^{13} \mathrm{AE}$ rates for trilaciclib in combination with etoposide/cisplatin were calculated by applying the relative risk reduction associated with trilaciclib to the rate of AEs without trilaciclib (pooled average from G1T28-05 and G1T28-02 clinical trials). ${ }^{43,44}$ In the second-line setting, AE rates for topotecan with or without trilaciclib were based on the trilaciclib G1T28-03 clinical trial. $^{45} \mathrm{AE}$ incidences for etoposide/carboplatin and etoposide/cisplatin were based on published literature. ${ }^{13,52} \mathrm{AE}$ rates for trilaciclib in combination with etoposide/carboplatin and etoposide/cisplatin were calculated by applying the relative risk reduction associated with trilaciclib to the rates of AEs without trilaciclib (from the G1T28-03 study). ${ }^{45}$

The average number of AEs (among patients who experienced at least $1 \mathrm{AE}$ ) in current and alternative scenarios were calculated based on the market share of available treatment options (Table 2). The data for trilaciclib were based on a pooled average from the G1T28-05 and G1T28-02 studies (first-line setting) and the G1T28-03 study (secondline setting).

Treatment and AE Costs. Treatment costs for chemotherapies considered in the model included acquisition and administration costs. Acquisition costs for each regimen per cycle were calculated by combining the published WAC for the recommended dose of each product (within the respective treatment regimen) from approved product labels and per recommended dosing schedule (Supplementary Table 1). ${ }^{53}$ Administration costs for treatment were based on published literature (Table 1).

The model assumed that all patients completed the recommended treatment regimens. A mean number of 4 cycles was assumed for the etoposide/carboplatin/atezolizumab regimen, consistent with the G1T28-05 study. ${ }^{44}$ A mean number of 5 cycles was assumed for the other chemotherapy regimens, as they are recommended for 4-6 cycles in international clinical guidelines ${ }^{6,7}$ and the G1T28-02 study followed patients until completion of chemotherapy as determined by the investigator (4-6 cycles; Supplementary Table 1).

The total cost of trilaciclib per course of chemotherapy was calculated by multiplying the WAC per dose of trilaciclib (\$1,417 per 300-mg vial or $\$ 2,834$ per dose) by the number of cycles in each chemotherapy regimen and by the number of doses required per cycle (Supplementary Table 1). ${ }^{54}$

The management costs for grade $3 / 4$ neutropenia, thrombocytopenia, and anemia were obtained from a retrospective claims-based analysis ${ }^{30}$ which assessed the incremental health care costs (ie, the difference in total cost of care for patients who experienced an $\mathrm{AE}$ compared with the total cost of care for patients who did not experience that $\mathrm{AE}$ ) associated with AEs in adult patients with cancer in the United States (Table 1). The management costs for FN were obtained from a retrospective claims-based analysis, ${ }^{21}$ which assessed clinical and economic risk and consequences of FN among patients with metastatic cancer in the United States.

All costs were adjusted to 2021 USD using the Consumer Price Index for medical care according to the US Bureau of Labor Statistics as of March 2021. ${ }^{29}$ Discounting was not applied, given the short survival for this population (up to 12-13 months). ${ }^{8,9}$

Inputs Related to Prophylactic Use of G-CSF. The NCCN guidelines for the management of neutropenia recommend the use of G-CSF as prophylaxis for any patients at high risk of FN (>20\%) and individualized consideration for patients at intermediate risk (10\%-20\%). ${ }^{33}$ The model estimated that prophylactic G-CSF was administered to $26 \%$ of patients with ES-SCLC receiving chemotherapy without trilaciclib, based on G1 Therapeutics market research. No direct data were available to calculate the reduction in G-CSF prophylactic use associated with the use of trilaciclib. Consequently, the value was based on reduction in use for any G-CSF (50\% reduction) as observed in a pooled analysis of clinical data for trilaciclib vs placebo in patients with ES-SCLC. 55

The average cost associated with prophylactic G-CSF per cycle was estimated to be $\$ 5,733$, which included the WAC (weighted average of branded G-CSF and biosimilars based on market share) and administration costs. ${ }^{53,56}$ The average number of G-CSF cycles per chemotherapy regimen (3.41) was based on a weighted average of the mean number of cycles for filgrastim (2.3) and pegfilgrastim (3.5). ${ }^{57}$ The average total cost of G-CSF per chemotherapy regimen was $\$ 19,548$. The therapeutic or rescue use of G-CSF (ie, use after the onset of neutropenia or FN) was assumed to be 


\section{TABLE 3 Total Budget Impact Over 5 Years Associated With the Introduction of Trilaciclib}

\begin{tabular}{l|c|c|c}
\hline & $\begin{array}{c}\text { Costs with current } \\
\text { scenario } \\
\text { (without trilaciclib) } \\
\mathbf{\$}\end{array}$ & $\begin{array}{c}\text { Costs with } \\
\text { alternative } \\
\text { scenario } \\
\text { (with trilaciclib) } \\
\mathbf{\$}\end{array}$ & $\begin{array}{c}\text { Cost } \\
\text { difference } \\
\mathbf{\$}\end{array}$ \\
\hline Trilaciclib & 0 & $3,704,199$ & $3,704,199$ \\
\hline Chemotherapy & $10,180,640$ & $10,180,640$ & 0 \\
\hline G-CSF prophylaxis & $1,532,595$ & $1,309,891$ & $-222,704$ \\
\hline AE management & $22,435,918$ & $18,153,170$ & $-4,282,748$ \\
\hline Neutropenia & $11,092,377$ & $8,824,574$ & $-2,267,803$ \\
\hline Febrile neutropenia & 731,642 & 574,521 & $-157,121$ \\
\hline Anemia & $3,801,868$ & $3,229,005$ & $-572,863$ \\
\hline Thrombocytopenia & $6,810,031$ & $5,525,070$ & $-1,284,961$ \\
\hline Total & $\mathbf{3 4 , 1 4 9 , 1 5 3}$ & $\mathbf{3 3 , 3 4 7 , 8 9 9}$ & $\mathbf{- 8 0 1 , 2 5 4}$ \\
\hline PMPM & $\mathbf{0 . 5 6 9}$ & $\mathbf{0 . 5 5 6}$ & $-\mathbf{0 . 0 1 3}$ \\
\hline $\begin{array}{l}\text { PPPM among patients eligible } \\
\text { for trilaciclib treatment }\end{array}$ & $\mathbf{1 , 8 8 7}$ & 1,843 & -44 \\
\hline AE & & & \\
\hline
\end{tabular}

$A E=$ adverse event; $G-C S F=$ granulocyte colony-stimulating factor; $P M P M=$ per member per month; PPPM = per-patient per-month. trilaciclib clinical trials (ie, first-line etoposide/carboplatin, first-line etoposide/carboplatin/atezolizumab, and second-line topotecan). The third assumed an administration cost for trilaciclib using a Current Procedural Terminology (CPT) code ( $\$ 77.86$ based on the Medicare National Fee Schedule for CPT 96365: IV infusion for therapy, prophylaxis, or diagnosis). ${ }^{58}$ The fourth considered the October 2021 CMS Medicare Part B reimbursement limit based on $2 \mathrm{Q}$ 2021 ASP for drug costs. ${ }^{48}$

\section{MODEL ASSUMPTIONS}

Several key assumptions were applied to this model. First, uptake of trilaciclib was based on internal forecasting and may differ from that observed in real-world clinical practice. Second, all patients were assumed to complete the recommended treatment regimens without interruptions, dose adjustments, or discontinuations. Third, chemotherapy treatment costs were fixed, and no differences were expected between the 2 treatment scenarios for this parameter. Fourth, trilaciclib had no effect on treatment response or survival, as no difference in progression-free survival or overall survival was observed in the trilaciclib clinical trials. ${ }^{43-45}$ Finally, only grade $\geq 3$ AEs (based on the National Cancer Institute's Common Terminology Criteria for Adverse Events) were included in the analysis, ${ }^{59}$ as these are most likely to require intervention and were considered to have the greatest impact on health and economic outcomes from a payer perspective.

\section{Results} Results were reported separately for the current (without trilaciclib) and alternative (with trilaciclib) scenarios, and the difference between the 2 scenarios. Total costs were presented in 4 subcategories: chemotherapy treatment cost, trilaciclib cost, $\mathrm{AE}$ management cost, and cost related to

\section{SENSITIVITY AND}

A 1-way DSA was performed to assess uncertainty around key model inputs. The following key model parameter WAC for trilaciclib, AE management cost, proportion of patients receiving prophylactic G-CSF without trilaciclib, reduction in prophylactic G-CSF use associated with trilaciclib, market uptake for trilaciclib, and population inputs related to patients eligible for trilaciclib.

Several scenario analyses were performed (Supplementary Table 2). The first considered a variation in the reduction in prophylactic use of G-CSF associated with trilaciclib outside the base-case value (ie, 0\%-100\%). The second was conducted to include only treatment regimens used in the

\section{BASE-CASE RESULTS}

Over a 5-year time horizon, an estimated total of 301 patients were eligible for treatment with trilaciclib per the labeled indication, comprising 


\section{FIGURE 2 One-Way DSA Results}

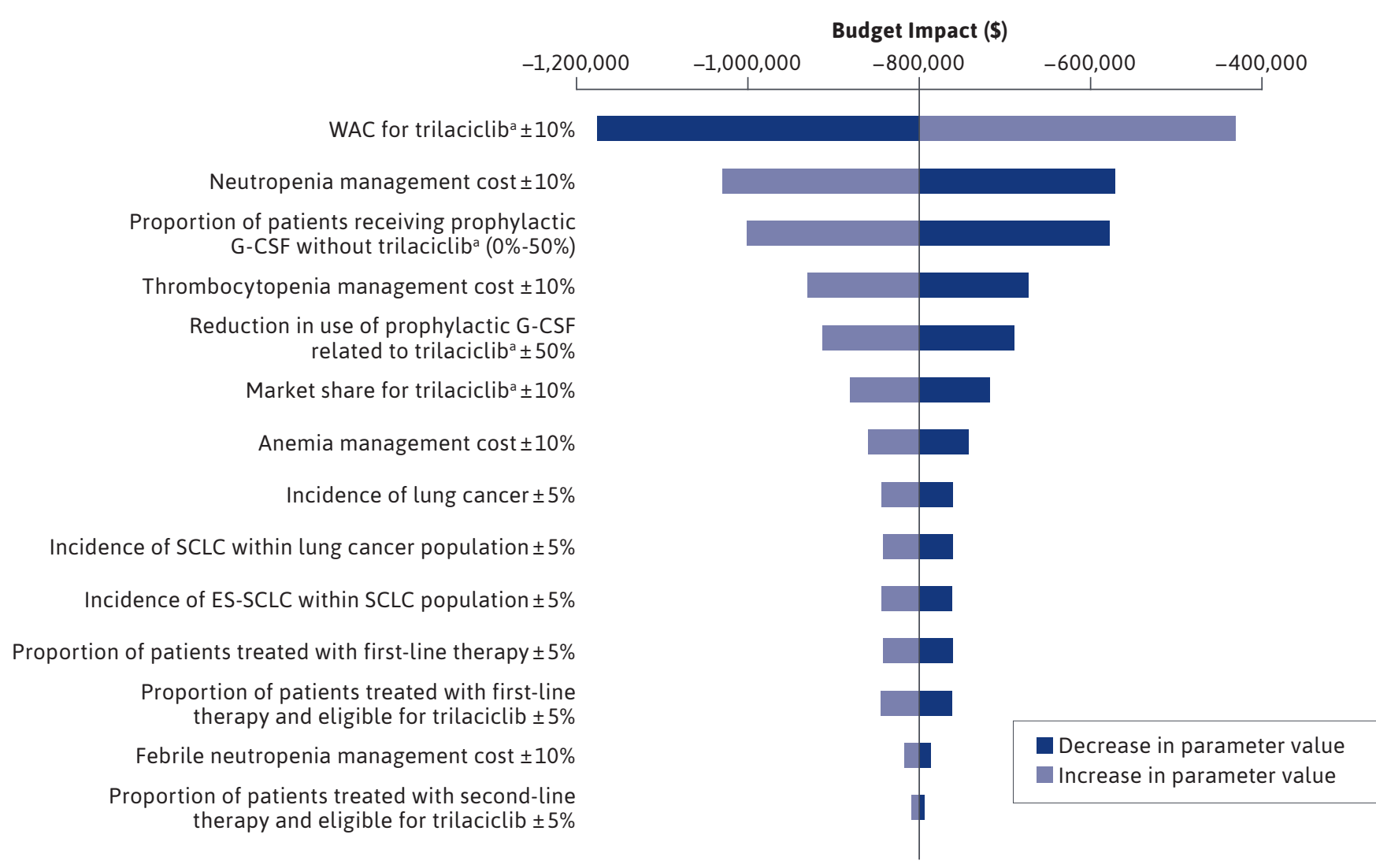

aFirst- and second-line settings combined.

$D S A=$ deterministic sensitivity analysis; $E S-S C L C=$ extensive-stage small cell lung cancer; $G-C S F=$ granulocyte colony-stimulating factor; $S C L C=s m a l l$ cell lung cancer; $W A C=$ wholesale acquisition cost.

239 and 62 in first- and second-line settings, respectively. The number of myelosuppressive AEs in the alternative scenario with trilaciclib were estimated to be fewer than in the current scenario without trilaciclib (events avoided over 5 years: 108 for neutropenia, 7 for FN, 23 for anemia, and 46 for thrombocytopenia; Supplementary Table 3).

In the base-case analysis, the total annual plan cost in the current scenario (without trilaciclib) was estimated to be $\$ 34,149,153$ for a 1 million-member health plan over 5 years. The total annual plan cost in the alternative scenario (with trilaciclib) was estimated to be $\$ 33,347,899$ over 5 years. The adoption of trilaciclib was therefore associated with cost savings of $\$ 801,254$ (translating to \$0.013 PMPM overall, and \$44 PPPM among the 301 patients eligible for trilaciclib treatment) over 5 years (Table 3 ).
A breakdown of the cost savings over 5 years in the alternative scenario shows that the cumulative $\$ 3,704,199$ acquisition cost for trilaciclib was offset by a net reduction in AE management cost $(\$ 4,282,748)$ and reduction in prophylactic G-CSF use (\$222,704).

The annual cost savings associated with trilaciclib were observed as early as year 1 (\$34,388 total, \$0.003 PMPM) and continued to accumulate over time. The total cumulative cost savings associated with treating patients with ES-SCLC receiving trilaciclib were \$155,828 (\$0.006 PMPM), $\$ 336,107$ (\$0.009 PMPM), \$555,651 (\$0.012 PMPM), and $\$ 801,254$ (\$0.013 PMPM) over 2, 3, 4, and 5 years, respectively (Supplementary Figure 1).

\section{SENSITIVITY AND SCENARIO ANALYSES}

Across all parameters adjusted in the 1-way DSA, the use of trilaciclib led to total cumulative cost savings ranging from 
$\$ 574,473$ to $\$ 1,171,673$ (\$0.010 to $\$ 0.020$ PMPM) over 5 years (Figure 2). The top 3 drivers of cost savings were the WAC for trilaciclib, management costs for neutropenia, and the prophylactic use of G-CSF without trilaciclib.

The scenario analysis that varied the percentage reduction in the prophylactic use of G-CSF associated with trilaciclib from $0 \%$ to $100 \%$ indicated a negligible effect: total cumulative cost savings with trilaciclib vs those without trilaciclib over 5 years ranged from $\$ 578,549$ (0\% reduction) to $\$ 1,023,958$ (100\% reduction), corresponding to $\$ 0.010$ to $\$ 0.017$ PMPM, and were like the base-case findings (Supplementary Table 2). The scenario analysis that included only treatment regimens collected in the trilaciclib clinical trials provided total cumulative cost savings over 5 years (\$0.015 PMPM) that were consistent with the base case (\$0.013 PMPM). The third scenario analysis, which assumed $\$ 77.65$ as the administration cost for trilaciclib under CPT code 96365, reported a total cumulative cost saving over 5 years of $\$ 0.012$ PMPM. The fourth scenario analysis, with October 2021 CMS Medicare Part B reimbursement limit based on 2Q 2021 ASP used for drug costs, reported a total cumulative cost saving over 5 years of \$0.008 PMPM (Supplementary Table 2).

\section{Discussion}

This is the first study to evaluate the financial impact of administering trilaciclib within 4 hours before the start of standard chemotherapy in patients with ES-SCLC enrolled in a health plan. The analysis shows that the introduction of trilaciclib translates into budget savings for a third-party payer when trilaciclib is used for its approved indication. Trilaciclib is the first approved myeloprotection therapy indicated to reduce the occurrence of CIM in adult patients. This BIM analysis estimated that trilaciclib would cumulatively reduce total annual management costs by $\$ 34,388$ (\$0.003 PMPM) at year 1 to $\$ 801,254$ (\$0.013 PMPM) at year 5 compared with treatment without trilaciclib.

The results of the model appear to be robust based on sensitivity and scenario analyses. The DSA showed that the WAC for trilaciclib was the biggest driver for the total cost savings associated with trilaciclib, with $\mathrm{AE}$ management costs and the use of prophylactic G-CSF in patients not receiving trilaciclib also key contributing drivers. The base case assumed a $50 \%$ reduction associated with trilaciclib in prophylactic G-CSF use, but even with $0 \%$ reduction in prophylactic G-CSF use, a cost saving with trilaciclib was still observed. Collectively, these data emphasize the financial impact to health care systems of having to manage CIM in patients with ES-SCLC.
Although prophylactic G-CSF use was identified as a cost driver, the intention of this analysis was not to provide a clinical or economic comparison of trilaciclib and G-CSF, which differ in their mechanism of action and intended use. By inducing cell-cycle arrest of hematopoietic stem and progenitor cells, trilaciclib proactively protects the bone marrow against chemotherapy-induced damage, ${ }^{34,42}$ and reduces the incidence of myelosuppression across multiple hematologic lineages (ie, neutrophils, red blood cells, and platelets). ${ }^{43-45}$ By contrast, G-CSF is used specifically for prevention or treatment of severe neutropenia and $\mathrm{FN} .{ }^{33,60}$ Moreover, although sometimes used prophylactically, G-CSF acts to stimulate stem cell growth and neutrophil production after the bone marrow is damaged.$^{61}$ Differences also exist in the eligibility of patients for treatment with trilaciclib and G-CSF, as trilaciclib is recommended to reduce the incidence of CIM in patients receiving platinum-/ etoposide- or topotecan-containing chemotherapy for ES-SCLC (an indication that does not involve prophylactic administration of G-CSF), while prophylactic use of G-CSF for FN is recommended when the risk of FN is high (>20\%) based on the chemotherapy regimen and patient risk factors. ${ }^{33}$ Of interest, a recent analysis of data pooled from 3 clinical trials of trilaciclib in patients with ES-SCLC showed that administering trilaciclib prior to chemotherapy significantly reduced the duration of severe neutropenia in cycle 1 and the occurrence of severe neutropenia across cycles 1 to 4 , regardless of G-CSF administration. ${ }^{62}$ The model included additional treatment regimens (etoposide/ cisplatin in the first-line setting, and etoposide/carboplatin and etoposide/cisplatin in the second-line setting) that were not included in trilaciclib clinical trials, with the intent to capture standard treatment options for ES-SCLC. The scenario analysis that only included treatment regimens collected in the trilaciclib trials provided cost savings (\$0.015 PMPM) that were consistent with the base case (\$0.013 PMPM).

\section{LIMITATIONS}

The BIM was developed based on several assumptions, as previously described. As the AE rates were based on the results from phase 2 trilaciclib clinical trials with relatively small sample sizes, there may be uncertainty regarding these estimates. Future studies are recommended to validate our results, ideally incorporating real-world evidence on $\mathrm{AE}$ incidence once sufficient data are available.

Although phase 3 data have traditionally been used to support marketing authorization and reimbursement of novel drugs, data from phase 2 trials are increasingly used as primary evidence for such purposes. ${ }^{63}$ By necessity, 
therefore, these data are subsequently used in economic models like ours. ${ }^{64}$

Health care resource utilization required for the administration of trilaciclib was not considered in the base-case analysis, given it is a 30-minute IV therapy (administered within 4 hours prior to chemotherapy) and can be performed during the same visit as that for administration of chemotherapy, for instance, with the administration of premedication. However, the scenario analysis that assumed administration using a CPT code reported total cumulative cost savings that were consistent with the base-case analysis results.

Cost inputs for $\mathrm{AE}$ management were obtained from large claimsbased studies involving multiple different oncology indications and therefore may not be specific to ES-SCLC. Despite having been tested in sensitivity analyses and yielding findings consistent with base-case results (with PMPM cost savings ranging from $\$ 0.008$ to $\$ 0.017$ in the sensitivity analysis vs $\$ 0.013$ in the base case), inputs specific to the setting of ES-SCLC are needed.

Finally, only grade $\geq 3$ AEs were considered within the AE management cost estimates, which may underestimate the health and economic impact of trilaciclib. Although there is no consensus on which AEs to include, economic models typically focus on grade $\geq 3 \mathrm{AEs},{ }^{65-68}$ as these are most likely to require resource utilization and have a meaningful impact on the results. Moreover, although lower-grade AEs may require medical services in some cases, consideration of all AEs would require additional model assumptions, ${ }^{30,65}$ which may reduce confidence in the model.

\section{Conclusions}

Trilaciclib is the first and, currently, the only multilineage, myeloprotective therapy proven to decrease the incidence of CIM for patients with ES-SCLC treated with a platinum-/ etoposide- or topotecan-containing regimen. Trilaciclib represents a new pharmacy expenditure when added to standard chemotherapy treatment regimens for ES-SCLC. However, the acquisition cost of trilaciclib to a third-party payer is projected to be offset by a reduction in the costs of managing AEs related to myelosuppression and those associated with prophylactic G-CSF, which are fewer with trilaciclib compared with chemotherapy alone.

The net financial impact of trilaciclib is estimated to be a budgetary cost saving when trilaciclib is used for its approved indication. The magnitude and rapidity of financial benefit will be affected by market uptake of trilaciclib and the incidence of myelosuppression unique to each payer's patient population.

\section{DISCLOSURES}

This research was funded by G1 Therapeutics, Inc., and implemented by ZS Associates, an independent consultancy that collated the model inputs and performed the budget impact analysis. The study sponsor was involved in the study design; collection, analysis, and interpretation of data; writing of the report; and the decision to submit the report for publication. The journal open access fee was funded by G1 Therapeutics, Inc.

Moran, Chioda, and Huang are employed by G1 Therapeutics, Inc. Chioda and Huang report stocks and stock options for G1 Therapeutics, Inc. Goyal and Deniz are employed by ZS Associates. Goyal reports consulting fees from G1 Therapeutics, Inc. Abraham reports consulting fees from Coherus, G1 Therapeutics, Inc. (unrelated to this study and manuscript), Mylan/ Viatris, and Sandoz and participation on a data safety monitoring board or advisory board for G1 Therapeutics, Inc. MacDonald reports consulting fees from Coherus, G1 Therapeutics, Inc. (unrelated to this study and manuscript), Mylan/Viatris, and Sandoz. Deniz reports no disclosures.
A synopsis of the current study was presented in poster format at the Virtual AMCP Annual Meeting, April 12-16, 2021.

\section{ACKNOWLEDGMENTS}

Medical writing assistance was provided by Tyrone Daniel, PhD, contracted by Alligent Europe (Envision Pharma Group) and funded by G1 Therapeutics, Inc.

\section{REFERENCES}

1. Siegel RL, Miller KD, Fuchs HE, Jemal A. Cancer statistics, 2021. CA Cancer J Clin. 2021;71(1):7-33.

2. American Cancer Society. Cancer facts \& figures 2021. Accessed April 1, 2021. https://www.cancer.org/ content/dam/cancer-org/research/ cancer-facts-and-statistics/annualcancer-facts-and-figures/2021/ cancer-facts-and-figures-2021.pdf

3. Wang S, Tang J, Sun T, et al. Survival changes in patients with small cell lung cancer and disparities between different sexes, socioeconomic statuses and ages. Sci Rep. 2017;7(1):1339.

4. Byers LA, Rudin CM. Small cell lung cancer: where do we go from here? Cancer. 2015;121(5):664-72.

5. Yang S, Zhang Z, Wang Q. Emerging therapies for small cell lung cancer. J Hematol Oncol. 2019;12(1):47.

6. Dingemans AMC, Früh M, Ardizzoni A, et al. Small-cell lung cancer: ESMO clinical practice guidelines for diagnosis, treatment and follow-up. Ann Oncol. 2021;32(7):839-53.

7. National Comprehensive Cancer Network. NCCN clinical practice guidelines in oncology. Small cell lung cancer. Version 3.2021. Accessed April 1, 2021. https://www.nccn.org/professionals/ physician gls/pdf/sclc.pdf

8. Horn L, Mansfield AS, Szczęsna A, et al; IMpower133 Study Group. First-line atezolizumab plus chemotherapy in extensive-stage small-cell lung cancer. N Engl J Med. 2018;379(23):2220-29. 
9. Paz-Ares L, Dvorkin M, Chen Y, et al; CASPIAN Investigators. Durvalumab plus platinum-etoposide versus platinumetoposide in first-line treatment of extensive-stage small-cell lung cancer (CASPIAN): a randomised, controlled, open-label, phase 3 trial. Lancet. 2019;394(10212):1929-39.

10. HYCAMTIN (topotecan hydrochloride) for injection. Prescribing information. GlaxoSmithKline; 2021. Accessed April 1, 2021. https://www.accessdata.fda.gov/ drugsatfda_docs/label/2010/020671s 016s017lbl.pdf

11. ZEPZELCA (lurbinectedin) for injection, for intravenous use. Prescribing information. Jazz Pharmaceuticals, Inc; 2021. Accessed April 1, 2021. https://www. accessdata.fda.gov/drugsatfda_docs/ label/2020/213702s000lbl.pdf

12. Noda K, Nishiwaki Y, Kawahara M, et al; Japan Clinical Oncology Group. Irinotecan plus cisplatin compared with etoposide plus cisplatin for extensive small-cell lung cancer. N Engl J Med. 2002;346(2):85-91.

13. Lara PN Jr, Natale R, Crowley J, et al. Phase III trial of irinotecan/cisplatin compared with etoposide/cisplatin in extensive-stage small-cell lung cancer: clinical and pharmacogenomic results from SWOG S0124. J Clin Oncol. 2009;27(15):2530-35.

14. Hanna N, Bunn PA Jr, Langer C, et al. Randomized phase III trial comparing irinotecan/cisplatin with etoposide/ cisplatin in patients with previously untreated extensive-stage disease small-cell lung cancer. J Clin Oncol. 2006;24(13):2038-43.

15. Han D, Wang G, Sun L, et al. Comparison of irinotecan/platinum versus etoposide/platinum chemotherapy for extensive-stage small cell lung cancer: a meta-analysis. Eur J Cancer Care (Engl). 2017;26(6).

16. TECENTRIQ (atezolizumab) for injection, for intravenous use. Prescribing information. Genentech, Inc; 2021. Accessed April 1, 2021. https://www.gene. com/download/pdf/tecentriq_prescribing.pdf
17. IMFINZI (durvalumab) for injection, for intravenous use. Prescribing information. AstraZeneca; 2021. Accessed April 1, 2021. https://den8dhaj6zs0e. cloudfront.net/50fd68b9-106b-4550b5d0-12b045f8b184/9496217c-08b3432b-ab4f-538d795820bd/9496217c08b3-432b-ab4f-538d795820bd viewable_rendition__v.pdf

18. von Pawel J, Jotte R, Spigel DR, et al. Randomized phase III trial of amrubicin versus topotecan as second-line treatment for patients with small-cell lung cancer. J Clin Oncol. 2014;32(35):4012-19.

19. Roe H, Lennan E. Role of nurses in the assessment and management of chemotherapy-related side effects in cancer patients. Nurs Res Rev. 2014;2014(4):103-15.

20. Crawford J, Dale DC, Lyman GH. Chemotherapy-induced neutropenia: risks, consequences, and new directions for its management. Cancer. 2004;100(2):228-37.

21. Weycker D, Li X, Edelsberg J, et al. Risk and consequences of chemotherapyinduced febrile neutropenia in patients with metastatic solid tumors. J Oncol Pract. 2015;11(1):47-54.

22. Lyman GH. Risks and consequences of chemotherapy-induced neutropenia. Clin Cornerstone. 2006;8(suppl 5):S12-18.

23. Kuter DJ. Managing thrombocytopenia associated with cancer chemotherapy. Oncology (Williston Park). 2015;29(4): 282-94.

24. Epstein RS, Aapro MS, Basu Roy UK, et al. Patient burden and real-world management of chemotherapy-induced myelosuppression: results from an online survey of patients with solid tumors. Adv Ther. 2020;37(8):3606-18.

25. Epstein RS, Basu Roy UK, Aapro M, et al. Cancer patients' perspectives and experiences of chemotherapy-induced myelosuppression and its impact on daily life. Patient Prefer Adherence. 2021;15:453-65.

26. Liou SY, Stephens JM, Carpiuc KT, Feng W, Botteman MF, Hay JW. Economic burden of haematological adverse effects in cancer patients: a systematic review. Clin Drug Investig. 2007;27(6):381-96.
27. Tai E, Guy GP, Dunbar A, Richardson LC. Cost of cancer-related neutropenia or fever hospitalizations, United States, 2012. J Oncol Pract. 2017;13(6):e552-61.

28. Weycker D, Hatfield M, Grossman A, et al. Risk and consequences of chemotherapy-induced thrombocytopenia in US clinical practice. BMC Cancer. 2019;19(1):151.

29. US Bureau of Labor Statistics. Consumer Price Index, March 2021. Accessed April 1, 2021. https://www.bls. gov/cpi/

30. Wong W, Yim YM, Kim A, et al. Assessment of costs associated with adverse events in patients with cancer. PLoS One. 2018;13(4):e0196007.

31. Bryer E, Henry D. Chemotherapyinduced anemia: etiology, pathophysiology, and implications for contemporary practice. Int J Clin Transfus Med. 2018;2018(6):21-31.

32. Gilreath JA, Rodgers GM. How I treat cancer-associated anemia. Blood. 2020;136(7):801-13.

33. National Comprehensive Cancer Network. NCCN clinical practice guidelines in oncology. Hematopoietic growth factors. Version 2.2021. Accessed April 1, 2021. https://www.nccn.org/professionals/physician_gls/pdf/growthfactors.pdf

34. Bisi JE, Sorrentino JA, Roberts PJ, Tavares FX, Strum JC. Preclinical characterization of G1T28: a novel CDK4/6 inhibitor for reduction of chemotherapyinduced myelosuppression. Mol Cancer Ther. 2016;15(5):783-93.

35. Lyman GH. Chemotherapy dose intensity and quality cancer care. Oncology (Williston Park). 2006;20(14 suppl 9):16-25.

36. Lyman GH. Impact of chemotherapy dose intensity on cancer patient outcomes. J Natl Compr Canc Netw. 2009;7(1):99-108. 
37. Morrow T, Siegel M, Boone S, Lawless G, Carter W. Chemotherapy dose intensity determination as a quality of care measure for managed care organizations in the treatment of earlystage breast cancer. Am J Med Qual. 2002;17(6):218-24.

38. Ottevanger PB, De Mulder PH. The quality of chemotherapy and its quality assurance. Eur J Surg Oncol. 2005;31(6):656-66.

39. Thomas K, Martin T, Gao A, Ahn C, Wilhelm H, Schwartz DL. Interruptions of head and neck radiotherapy across insured and indigent patient populations. J Oncol Pract. 2017;13(4):e319-28.

40. Claessens AKM, Erdkamp FLG, Lopez-Yurda M, et al; Dutch Breast Cancer Research Group (BOOG). Secondary analyses of the randomized phase III Stop\&Go study: efficacy of second-line intermittent versus continuous chemotherapy in HER2-negative advanced breast cancer. Acta Oncol. 2020;59(6):713-22.

41. Bese NS, Sut PA, Ober A. The effect of treatment interruptions in the postoperative irradiation of breast cancer. Oncology. 2005;69(3):214-23.

42. He S, Roberts PJ, Sorrentino JA, et al. Transient CDK4/6 inhibition protects hematopoietic stem cells from chemotherapy-induced exhaustion. Sci Transl Med. 2017;9(387):eaal3986.

43. Weiss JM, Csoszi T, Maglakelidze M, et al; G1T28-02 Study Group.

Myelopreservation with the CDK4/6 inhibitor trilaciclib in patients with small-cell lung cancer receiving first-line chemotherapy: a phase Ib/ randomized phase II trial. Ann Oncol. 2019;30(10):1613-21.

44. Daniel D, Kuchava V, Bondarenko I, et al. Trilaciclib prior to chemotherapy and atezolizumab in patients with newly diagnosed extensive-stage small cell lung cancer: a multicentre, randomised, double-blind, placebo-controlled phase II trial. Int J Cancer. 2020;148(10):2557-70.
45. Hart LL, Ferrarotto R, Andric ZG, et al. Myelopreservation with trilaciclib in patients receiving topotecan for small cell lung cancer: results from a randomized, double-blind, placebo-controlled phase II study. Adv Ther. 2021;38(1):350-65.

46. COSELA (trilaciclib) for injection, for intravenous use. Prescribing information. G1 Therapeutics, Inc; 2021. Accessed April 1, 2021. https://www. accessdata.fda.gov/drugsatfda_docs/ label/2021/214200s000lbl.pdf

47. Sullivan SD, Mauskopf JA, Augustovski F, et al. Budget impact analysis-principles of good practice: report of the ISPOR 2012 Budget Impact Analysis Good Practice II Task Force. Value Health. 2014;17(1):5-14.

48. Centers for Medicare \& Medicaid Services. 2021 Medicare Parts A \& B premiums and deductibles. Accessed November 24, 2021. https://www.cms. gov/medicare/medicare-part-b-drugaverage-sales-price/2021-asp-drugpricing-files

49. de Groot PM, Wu CC, Carter BW, Munden RF. The epidemiology of lung cancer. Transl Lung Cancer Res. 2018;7(3):220-33.

50. Worldometers. US Population 2018. 2019. Accessed December 1, 2019. https://www.worldometers.info/ world-population/us-population/

51. American Cancer Society. Small cell lung cancer stages. 2019. Accessed April 1, 2021. https://www.cancer.org/cancer/ lung-cancer/detection-diagnosis-staging/staging-sclc.html

52. Baize N, Monnet I, Greillier L, et al; Groupe Français de Pneumo-Cancérologie 01-13 Investigators. Carboplatin plus etoposide versus topotecan as secondline treatment for patients with sensitive relapsed small-cell lung cancer: an openlabel, multicentre, randomised, phase 3 trial. Lancet Oncol. 2020;21(9):1224-33.

53. Drugs.com. 2020. Accessed December 1, 2019. http://www.drugs.com

54. IBM. Micromedex. RED BOOK online. Database. 2021. Accessed March 19, 2021. https://www.ibm.com/products/ micromedex-red-book
55. Weiss J, Goldschmidt J, Andric Z et al. Effects of trilaciclib on chemotherapy-induced myelosuppression and patient-reported outcomes in patients with extensive-stage small cell lung cancer: pooled results from three phase II randomized, double-blind, placebocontrolled studies. Clin Lung Cancer. 2021;22(5):449-60.

56. Eldar-Lissai A, Cosler LE, Culakova E, Lyman GH. Economic analysis of prophylactic pegfilgrastim in adult cancer patients receiving chemotherapy. Value Health. 2008;11(2):172-79.

57. Naeim A, Henk HJ, Becker L, et al. Pegfilgrastim prophylaxis is associated with a lower risk of hospitalization of cancer patients than filgrastim prophylaxis: a retrospective United States claims analysis of granulocyte colony-stimulating factors (G-CSF). BMC Cancer. 2013;13:11.

58. Centers for Medicare \& Medicaid Services. Medicare national fee schedule 2021. Accessed July 1, 2021. https://www.cms.gov/Medicare/ Medicare-Fee-for-Service-Payment/ PhysicianFeeSched

59. National Cancer Institute. Common terminology criteria for adverse events (CTCAE) v4.03. Accessed November 22, 2021. https://evs.nci.nih.gov/ftp1/ CTCAE/CTCAE_4.03/CTCAE_4.03_201006-14_QuickReference_5x7.pdf

60. Klastersky J, de Naurois J, Rolston K, et al; ESMO Guidelines Committee. Management of febrile neutropaenia: ESMO clinical practice guidelines. Ann Oncol. 2016;27(suppl 5):v111-18.

61. Bendall LJ, Bradstock KF. G-CSF: from granulopoietic stimulant to bone marrow stem cell mobilizing agent. Cytokine Growth Factor Rev. 2014;25(4):355-67.

62. Ferrarotto R, Anderson I, Medgyasszay B, et al. Trilaciclib prior to chemotherapy reduces the usage of supportive care interventions for chemotherapy-induced myelosuppression in patients with small cell lung cancer: pooled analysis of three randomized phase 2 trials. Cancer Med. 2021;10(17):5748-56. 
63. Li YYR, Mai H, Trudeau ME, et al. Reimbursement recommendations for cancer drugs supported by phase II evidence in Canada. Curr Oncol. 2020;27(5):e495-500.

64. Bennett CL, Lane D, Stinson T, et al. Economic analysis of amifostine as adjunctive support for patients with advanced head and neck cancer: preliminary results from a randomized phase II clinical trial from Germany. Cancer Invest. 2001;19(2):107-13.

65. Wong W, Carlson J, Cloutier M. Estimating the costs of adverse events in economic models: is there a "right" approach? Value Outcomes Spotlight. 2019;5(3):27-29.
66. Cai B, Zhou ZY, Xue W, et al. Budget impact of capmatinib for adults with metastatic non-small cell lung cancer harboring a MET exon 14 skipping mutation in the United States. J Med Econ. 2021;24(1):131-39.

67. Bharmal M, Kearney M, Zheng Y, Phatak H. Budget impact model of avelumab in patients with metastatic merkel cell carcinoma in the US. Clinicoecon Outcomes Res. 2019;11:349-59.

68. Proudman D, Miller A, Nellesen D, et al. Financial implications of avapritinib for treatment of unresectable gastrointestinal stromal tumors in patients with a PDGFRA exon 18 variant or after 3 previous therapies in a hypothetical US health plan. JAMA Netw Open. 2020;3(11):e2025866.
69. Shiozawa T, Sekine I, Aida Y, et al. Rechallenge with first-line platinum chemotherapy for sensitive-relapsed small-cell lung cancer. Case Rep Oncol. 2018;11(3):622-32.

70. Duh MS, Hackshaw MD, Ivanova JI, et al. Costs associated with intravenous cancer therapy administration in patients with metastatic soft tissue sarcoma in a US population. Sarcoma. 2013;2013:947413. 